\title{
PROPAGACIÓN DE Tabebuia Donnell-Smithii Rose (GUAYACÁN BLANCO) UTILIZANDO HORMONAS DE ENRAIZAMIENTO
}

\author{
PROPAGATION OF Tabebuia Donnell-Smithii Rose (WHITE GUAYACÁN) \\ USING ROOTINGS HORMONES
}

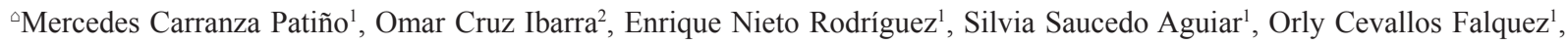
Ariel Escobar Troya ${ }^{1}$, Ximena Reyes Chancay ${ }^{1}$, Jaime Morante Carriel ${ }^{1}$

${ }^{I}$ Dirección de Investigación Cientifica y Tecnológica. Laboratorio de Biotecnología. Universidad Técnica Estatal de Quevedo. Campus Ing. Manuel Haz Álvarez, $\mathrm{km} 1.5$ vía a Santo Domingo de los Tsáchilas. C. P. 73.Quevedo,Los Ríos, Ecuador. ${ }^{\triangleright}$ susycespinosa@yahoo.es

${ }^{2}$ Facultad de Ciencias Ambientales, Universidad Técnica Estatal de Quevedo. Campus Ing. Manuel Haz Álvarez, km 1.5 vía a Santo Domingo de los Tsáchilas. C. P. 73. Quevedo, Los Ríos, Ecuador.
\end{abstract}

\begin{abstract}
Resumen
T a amplia distribución y abundancia natural de LTabebuia donnell-smithii (guayacán blanco) se ha visto reducida por la tala excesiva de los árboles, lo que ha ocasionado la desaparición de genotipos valiosos así como disminución de las poblaciones naturales, existiendo además carencia de alternativas de producción de plantas a gran escala. Se hace por tanto necesario orientar la investigación a establecer una técnica para la propagación vegetativa de guayacán blanco utilizando fitorreguladores. La metodología se basó en el uso de las hormonas de enraizamiento ácido naftalenacético (ANA) y ácido indolbutírico (AIB), en concentraciones de $0,1,500$ y $2,000 \mathrm{mg} \mathrm{kg}^{-1}$ en sustratos turba y arena. Las yemas apicales fueron colocadas en una cámara húmeda en condiciones de invernadero. Se aplicó un diseño completamente al azar (DCA) en un arreglo factorial 2 sustratos $\mathrm{x} 3$ dosis de hormona ANA x 3 dosis de hormona AIB, con cuatro repeticiones y cuatro unidades de observación. A los 45 días se evaluó el porcentaje de sobrevivencia y el enraizamiento, el número de raíces, la longitud de la raíz mayor, el número de brotes, la longitud de brotes, y el vigor. Los resultados no mostraron diferencias significativas entre los distintos tratamientos para todas las variables, sin embargo para el efecto simple e interacciones se observó diferencias para las variables evaluadas, siendo el mejor sustrato turba y las concentraciones óptimas de auxinas de 0 a $1,500 \mathrm{mg} \mathrm{Kg}^{-1}$. Se concluye que el guayacán blanco es una especie de fácil enraizamiento, ya que con y sin la aplicación de hormonas enraizadoras en el sustrato turba y arena se logró la obtención de clones, disminuyendo el tiempo de obtención de nuevas plantas.
\end{abstract}

Palabras clave: Ácido naftalenacético, ácido indolbutírico y propagación asexual.

Recibido: 28-Enero-2012. Recibido en forma corregida: 30-Agosto-2012. Aceptado: 27-Noviembre-2012.

Publicado como ARTÍCULO CIENTÍFICO en Ciencia y Tecnología 5(2): 17-26. 2012
Abstract

$\mathrm{T}$ The wide distribution and natural abundance of Tabebuia donnell-smithii (white guayacán) has been reduced by overcutting of the trees, which has caused the disappearance of valuable genotypes as well as natural population decline, also, there is lack of alternatives in production of large-scale plants. Hence, it is necessary to orientate the research toward to establish a technique for the vegetative propagation of white guayacán using phytoregulators. The methodology was based on the use of rooting hormones, naphthaleneacetic acid (NAA) and indole butyric acid (IBA) at concentrations of $0,1,500$ and $2,000 \mathrm{mg} \mathrm{kg}^{-1}$ in peat and sand substrates. Apical buds were placed in a moist chamber under greenhouse conditions. We used a completely randomized design (DCA) in a factorial $2 \times 3$ substrates ANA hormone dose $\mathrm{x} 3$ doses of hormone AIB. With four replications and four observation units. At 45 days were evaluated: survival and rooting percentage, number of roots, greater root length, number of shoots, shoot length and vgor. The results did not show significant differences between treatments for all variables, however for the simple effect and interactions were observed differences for the variables evaluated, being the best peat substrate and optimal concentrations of auxins from $0-1,500 \mathrm{mg} \mathrm{kg}^{-1}$. In conclusion, the results showed that white guayacán is a kind of easy rooting inasmuch as with and without the application of hormones in the peat and sand substrate, it was achieved the obtaining of clones, reducing the time to obtain new plants.

Key words: Asexual propagation, indolebutyric acid and naphthaleneacetic acid. 


\section{INTRODUCCIÓN}

E l Ecuador se caracteriza por tener una vasta riqueza de recursos naturales y diversidad de ecosistemas boscosos, pero también por los altos índices de deforestación. Cuenta con aproximadamente 11.5 millones de hectáreas cubiertas de bosques naturales, que representan el $42 \%$ del área total del territorio, el $37 \%$ de los bosques están declarados como Reservas Naturales Protegidas, que equivale al 18\% del territorio nacional (Martínez, 1997, citado por el Ministerio de Relaciones Exteriores, Comercio e Integración, 2010). América Latina se sitúa con una de las más altas tasas de deforestación; entre $1.2 \%$ y $1.7 \%$ anual (Martínez, 1997, citado por el Ministerio de Relaciones Exteriores, Comercio e Integración, 2010). A pesar de esto el panorama de la deforestación a nivel mundial de acuerdo a la FAO, ha disminuido a lo largo de los últimos 10 años (FAO, 2010).

Tabebuia donnell-smithii (guayacán blanco), es conocido comúnmente como primavera, posee una gran distribución y abundancia natural, la cual se ha visto reducida debido a la tala excesiva, provocada por la gran demanda de madera existente en el mercado, donde alcanza un gran precio debido a la calidad de su madera, reportando innumerables usos entre ellos muebles, molduras y chapa decorativa. Este árbol de gran tamaño además de cultivarse para la producción de madera, cuyo grado de rendimiento es extremadamente bueno, se lo hace también como un árbol de ornamento, en parte debido a su impresionante despliegue estacional de flores amarillas (John, 1989). El rango nativo de esta especie se extiende desde México a través de Guatemala, El Salvador y el norte y centro de Honduras. Fuera de este rango ha sido evaluada como árbol maderable en Costa Rica, Hawai, Puerto Rico y Ecuador (Vozzo, 2010).

Se propaga por semilla con un porcentaje de germinación promedio de 47\% Vozzo (2010). Este tipo de propagación en función de la recombinación genética no garantiza un individuo con las mismas características seleccionadas de las plantas matrices (Ono, 1992, citado por Wendling, 2004). Diferentes estrategias de propagación permiten la obtención y multiplicación clonal, entre ellos la micropropagación in vitro, el desarrollo de semillas artificiales que implica el empleo de embriones somáticos encapsulados en una testa sintética protectora, obtención de embriones somáticos, las mismas que requieren de mano de obra calificada, alta inversión en las instalaciones especializadas, encareciendo el costo de la planta (Mrooginski y Roca, 1991).

La principal ventaja asociada a la utilización de la propagación vegetativa se encuentra en el campo del mejoramiento genético, debido a que permite transferir aquellas características que por su baja heredabilidad no se traspasan eficientemente a la descendencia por vía sexual, mientras no se utilicen técnicas especiales como polinizaciones controladas o huertos biclonales (Gutiérrez y Chung, 1994).

Esta técnica permite obtener mayores retornos por ganancias en la calidad y la uniformidad de las plantaciones, siendo este el principal motivo de su utilización (Monteuuis et al., 1998) (Koenig y Melchior, 1978), La propagación vegetativa puede ser de interés para plantas que son difíciles de enraizar (Serrano et al., 1988), además puede mostrar incrementos sustanciales de los índices de multiplicación en periodos de tiempo relativamente cortos demostrando tener gran importancia en la propagación asexual o vegetativa de plantas leñosas (Santelices y Cabello, 2006), sin embargo según Navarro et al. (2006) hay que analizar las interacciones entre la morfología de las plantas y otros aspectos de la repoblación.

La morfología de una planta cultivada en contenedor en un vivero forestal es el resultado de las características genéticas de las plantas, las condiciones ambientales del vivero y las prácticas de cultivo empleadas, tales como la fecha de siembra, la densidad de cultivo, el tipo de contenedor, el grado de sombreo, el régimen de fertilización y riego, las podas aéreas, entre otros. (Mexal y Landis, 1990, citado por Navarro et al., 2006).

Surge por tanto la necesidad de estudiar nuevas formas de propagación vegetativa rescatando genotipos valiosos, con el uso de auxinas, hormonas que regulan múltiples procesos fisiológicos en las plantas, entre ellos la iniciación de raíces. Por otro lado el sustrato tiene un efecto importante en el éxito del enraizamiento, y debe ser considerado como parte integral de cualquier sistema de propagación (Hartmann et al., 1992). Un aspecto importante en los cultivos lo constituye precisamente garantizar los requerimientos nutrimentales, proceso en el cual interactúan activamente las plantas, el suelo y las fuentes externas de suministro de nutrientes (Morales et al., 2011).

Con la finalidad de apoyar procesos que contribuyan a la recuperación de los bosques y por lo antes descrito, resulta imprescindible la búsqueda de una técnica de propagación eficiente para guayacán blanco. Por ello esta investigación tuvo como objetivo establecer una metodología para la propagación asexual de guayacán blanco, mediante la aplicación de hormonas de enraizamiento ANA y AIB en distintas concentraciones y sustratos (arena y turba). 


\section{Materiales Y MÉTODOS}

E sta investigación se llevó a cabo en el invernadero del Laboratorio de Biotecnología de la Universidad Técnica Estatal de Quevedo, ubicado en el km 1.5 vía Santo Domingo de los Tsáchilas.

El invernadero está cubierto por una malla de sombra al $70 \%$, situadas horizontal y verticalmente, cubriendo la estructura metálica de $3 \mathrm{~m}$ de alto $\times 7 \mathrm{~m}$ largo y 3 de ancho, permitiendo la disminución en la radiación solar y/o regulando la evapo-transpiración. Dentro de ésta área la temperatura promedio es de $24^{\circ}$ $\mathrm{C}$, la humedad relativa de $88 \%$, y la intensidad lumínica es 8 horas luz 16 oscuridad.

Preparación de hormonas. Se pesaron las diferentes concentraciones de hormonas, tanto el ácido indol butírico (Indol-3-butyric acid) AIB y el ácdio naftalen acético (naphthaleneacetic acid) de la marca sigma con $97 \%$ y $>98 \%$ de pureza respectivamente, de acuerdo a cada tratamiento en la relación $1 \mathrm{~g}^{-1} \mathrm{~kg}^{-1}$, así para la concentración $1,000 \mathrm{mg} \mathrm{kg}^{-1}$ se pesó $0.01 \mathrm{~g}$ de hormona que se disolvió con $3 \mathrm{~mL}$ de hidróxido de sodio $(\mathrm{NaOH})$ (Ramos et al., 2006), definiéndose el peso de las otras concentraciones por regla de tres simple. Posteriormente se mezclaron con $10 \mathrm{~g}$ de silicato de magnesio $\mathrm{Mg}_{3}$ $\left(\mathrm{SiO}_{10}\right)(\mathrm{OH})_{2}$ (talco), y alcohol al $70 \%$ hasta formar una masa pastosa, una vez mezclado se la extendió en un recipiente de aluminio plano a temperatura ambiente por 24 horas, luego fueron envasadas en frascos de vidrio y rotuladas. El tratamiento testigo $\left(0 \mathrm{mg} \mathrm{kg}^{-1}\right)$ solo recibió la aplicación del talco.

Sustratos empleados. Se utilizó arena de río formada por pequeños granos de alrededor de 0.05 a $2 \mathrm{~mm}$ de diámetro, virtualmente no contiene nutrientes minerales y no tiene capacidad amortiguadora (Buffer) o capacidad de intercambio catiónico (Hartmann et al., 1992). La turba utilizada fue Biolan de AGRIPAC con una porosidad del $78 \%$ y un $\mathrm{pH}$ de 6.50 , los mismos que fueron desinfectados con fungicida (Captan 80) ingrediente activo captan en proporciones de $2 \mathrm{~g} \mathrm{~L}^{-1}$ cada $4.54 \mathrm{~kg}$ de sustrato, ocho días previos al establecimiento del experimento para prevenir posible ataque de hongos. Los sustratos fueron colocados en bandejas germinadoras de 24 pocillos de $6 \mathrm{~cm}$ de diámetro y 15 $\mathrm{cm}$ de profundidad, los mismos que fueron enriquecidos con $40 \mathrm{Kg}^{-1}$ de macro y micronutrientes propuestos por Murashige y Skoog (1962) al 100\% de la concentración de las sales, a través del empleo de una bomba manual, acción que fue realizada el día de la siembra.

Material vegetativo y siembra. Se utilizaron miniestacas de la parte apical de 6 a $8 \mathrm{~cm}$ de longitud provenientes de plántulas de seis semanas de edad, con características fisiológicas y morfológicas propicias. Cada sección vegetal a propagar de los brotes principales de cada plántula de guayacán blanco fue cortado con una tijera podadora, previamente desinfectada con alcohol al $70 \%$, las hojas de los brotes fueron cortadas entre el 50 y $60 \%$ dependiendo del tamaño; posteriormente fueron sumergidas en una solución fúngica de Benomyl al 2\% (ingrediente activo) para evitar la deshidratación y contaminación de las mismas; antes de proceder a la siembra se le realizó un nuevo corte en forma de bisel en la base, lugar en donde se colocó la solución de enraizamiento, eliminando luego el exceso, en el caso del testigo solo se colocó el polvo sin el suplemento hormonal, inmediatamente se procedió a colocarlas en el sustrato hasta una profundidad de tres a cuatro centímetros, de acuerdo a cada tratamiento. Posteriormente se cubrió con plástico de polietileno a un altura de $0.8 \mathrm{~m}$ formando una cámara húmeda dentro del umbráculo el mismo que estaba protegido por zarán al $30 \%$, evitando el efecto directo de la luz sobre las estacas. El riego se realizó periódicamente con un aspersor manual en horas de la mañana para mantener la humedad relativa y evitar la desecación de los brotes.

Aclimatación. Al terminar el proceso de enraizamiento en la cámara de polietileno, que duró 45 días, se extrajeron las plantas del sustrato, las mismas fueron lavadas con agua corriente a fin de visualizar mejor las raíces, luego de la evaluación fueron trasferidas a fundas de polietileno $(8 \times 12 \mathrm{~cm})$ que contenían tierra de monte, previamente desinfectada $2 \mathrm{~g} \mathrm{~L}^{-1}$ cada 4.54 $\mathrm{kg}$ de sustrato, para luego pasar a la aclimatación. Este proceso consistió en destapar la cámara húmeda por una hora progresiva cada día, durante ocho días; es decir se quitó el plástico de polietileno procediendo también a dar riego.

Variables evaluadas. A los 45 días se evaluó el porcentaje de sobrevivencia y enraizamiento, para lo cual se contó el número de estacas (vivas o enraizadas), dividido para el total de estacas establecidas inicialmente, dependiendo del caso multiplicado por 100. El número de raíces mayor a $1 \mathrm{~mm}$ y número de brotes, se evaluó contando cada uno de ellos. Para la longitud de raíz se midió la raíz principal de cada uno de los brotes; la longitud de brotes se evaluó midiendo el brote mayor en cada planta, en ambos casos se utilizó un calibrador Vernier.

Análisis estadístico. Para analizar las condiciones experimentales, se contempló tres factores: Tipos de sustratos (arena y turba), concentración de hormona ANA $\left(1,500,2,000\right.$ y $\left.0 \mathrm{mg} \mathrm{Kg}^{-1}\right)$ y concentración de 
hormona $\mathrm{AIB}\left(1,500,2,000\right.$ y $\left.0 \mathrm{mg} \mathrm{Kg}^{-1}\right)$, dando la combinación de ellos 18 tratamientos, formados por cuatro repeticiones y cuatro unidades de observaciones, cada uno en forma completamente al azar (Cuadro 1). Los datos de cada una de las variables se sometieron a un análisis de varianza ANDEVA y separación de medias al 95\% de probabilidad, el mismo se realizó con ayuda del programa estadístico MSTAC. Para establecer diferencias estadísticas entre tratamientos se efectuó la prueba de rangos múltiples de Tukey ( $\mathrm{p} \geq 0.05)$. Los datos con valores cero fueron transformados con la fórmula: $\sqrt{ }(x+0.5)$

Cuadro 1. Tratamientos utilizados en la propagación asexual de Tabebuia donnell-Smithii Rose (guayacán blanco)

\begin{tabular}{|c|c|c|}
\hline $\mathbf{N}^{\circ}$ Tratamientos & & Descripción \\
\hline 1 & A1B1C1 & Turba $+1,500 \mathrm{mg} \mathrm{kg}^{-1} \mathrm{ANA}+1,500 \mathrm{mg} \mathrm{kg}^{-1} \mathrm{AIB}$ \\
\hline 2 & $\mathrm{~A} 1 \mathrm{~B} 1 \mathrm{C} 2$ & Turba $+1,500 \mathrm{mg} \mathrm{kg}^{-1} \mathrm{ANA}+2,000 \mathrm{mg} \mathrm{kg}^{-1} \mathrm{AIB}$ \\
\hline 3 & $\mathrm{~A} 1 \mathrm{~B} 1 \mathrm{C} 3$ & Turba $+1,500 \mathrm{mg} \mathrm{kg}^{-1}$ ANA \\
\hline 4 & $\mathrm{~A} 1 \mathrm{~B} 2 \mathrm{C} 1$ & Turba $+2,000 \mathrm{mg} \mathrm{kg}^{-1} \mathrm{ANA}+1,500 \mathrm{mg} \mathrm{kg}^{-1} \mathrm{AIB}$ \\
\hline 5 & $\mathrm{~A} 1 \mathrm{~B} 2 \mathrm{C} 2$ & Turba $+2,000 \mathrm{mg} \mathrm{kg}^{-1} \mathrm{ANA}+2,000 \mathrm{mg} \mathrm{kg}^{-1} \mathrm{AIB}$ \\
\hline 6 & $\mathrm{~A} 1 \mathrm{~B} 2 \mathrm{C} 3$ & Turba $+2,000 \mathrm{mg} \mathrm{kg}^{-1} \mathrm{ANA}$ \\
\hline 7 & $\mathrm{~A} 1 \mathrm{~B} 3 \mathrm{C} 1$ & Turba $+1,500 \mathrm{mg} \mathrm{kg}^{-1}$ AIB \\
\hline 8 & $\mathrm{~A} 1 \mathrm{~B} 3 \mathrm{C} 2$ & Turba $+2,000 \mathrm{mg} \mathrm{kg}^{-1} \mathrm{AIB}$ \\
\hline 9 & $\mathrm{~A} 1 \mathrm{~B} 3 \mathrm{C} 3$ & Turba $+0 \mathrm{mg} \mathrm{kg}^{-1} \mathrm{AIB}$ \\
\hline 10 & $\mathrm{~A} 2 \mathrm{~B} 1 \mathrm{C} 1$ & Arena $+1,500 \mathrm{mg} \mathrm{kg}^{-1} \mathrm{ANA}+1,500 \mathrm{mg} \mathrm{kg}^{-1} \mathrm{AIB}$ \\
\hline 11 & $\mathrm{~A} 2 \mathrm{~B} 1 \mathrm{C} 2$ & Arena $+1,500 \mathrm{mg} \mathrm{kg}^{-1} \mathrm{ANA}+2,000 \mathrm{mg} \mathrm{kg}^{-1} \mathrm{AIB}$ \\
\hline 12 & $\mathrm{~A} 2 \mathrm{~B} 1 \mathrm{C} 3$ & Arena $+1,500 \mathrm{mg} \mathrm{kg}^{-1} \mathrm{ANA}$ \\
\hline 13 & $\mathrm{~A} 2 \mathrm{~B} 2 \mathrm{C} 1$ & Arena $+2,000 \mathrm{mg} \mathrm{kg}^{-1} \mathrm{ANA}+1,500 \mathrm{mg} \mathrm{kg}^{-1} \mathrm{AIB}$ \\
\hline 14 & $\mathrm{~A} 2 \mathrm{~B} 2 \mathrm{C} 2$ & Arena $+2,000 \mathrm{mg} \mathrm{kg}^{-1} \mathrm{ANA}+2,000 \mathrm{mg} \mathrm{kg}^{-1} \mathrm{AIB}$ \\
\hline 15 & $\mathrm{~A} 2 \mathrm{~B} 2 \mathrm{C} 3$ & Arena $+2,000 \mathrm{mg} \mathrm{kg}^{-1} \mathrm{ANA}$ \\
\hline 16 & $\mathrm{~A} 2 \mathrm{~B} 3 \mathrm{C} 1$ & Arena $+1,500 \mathrm{mg} \mathrm{kg}^{-1} \mathrm{AIB}$ \\
\hline 17 & $\mathrm{~A} 2 \mathrm{~B} 3 \mathrm{C} 2$ & Arena $+2,000 \mathrm{mg} \mathrm{kg}^{-1} \mathrm{AIB}$ \\
\hline 18 & $\mathrm{~A} 2 \mathrm{~B} 3 \mathrm{C} 3$ & Arena $+0 \mathrm{mg} \mathrm{kg}^{-1} \mathrm{AIB}$ \\
\hline
\end{tabular}

\section{RESUlTADOS Y DISCUSIÓN}

Efecto simple. El análisis demostró, que existieron diferencias significativas para los tres factores, A, B y $\mathrm{C}$, los mejores promedios obtenidos para la mayoría de las variables fueron en el factor A, el sustrato turba (A1), en el factor B y C la concentración 1,500 $\mathrm{mg} \mathrm{kg}^{-1}$ y el testigo (B1) (C1) (Cuadro 2).

En la variable porcentaje de sobrevivencia en los factores B (hormona ANA) y C (hormona AIB) los niveles B3 (92.71\%), C3 (89.58\%) y C1 (85.42\%) fueron estadísticamente superiores, lo que demuestra que el guayacán blanco puede propagarse a partir de estacas sin dificultad. Estos resultados son similares a los obtenidos por Cruz et al. (2008) quienes obtuvieron un porcentaje de sobrevivencia de 93.33 en (Fernán Sánchez) en brotes de Chlorophora tinctoria (L) Gaud (moral fino). Según Santelices (1993) citado por Santelices y Cabello (2006) uno de los parámetros más importantes a medir en reproducción vegetativa es la sobrevivencia de las micro estacas, ya que para obtener un enraizamiento satisfactorio, es esencial la sobrevivencia de un gran número del material vegetal.

En el efecto simple, la variable porcentaje de enraizamiento mostró diferencias significativas frente a los factores A, B y C, con promedios altos, observados en los niveles A1(84.72), B1 (83.33) y B3 (86.46), C1 (84.38) y C3 (84.38) (Cuadro 2). Estos resultados son similares a los obtenidos por Ramos et al. (2006) quienes obtuvieron $83.30 \%$ en Chlorophora tinctoria $\mathrm{L}$. Gaud en la concentración 1,000 mg Kg-1 ANA + 1,000 mg $\mathrm{Kg}^{-1}$ AIB, también superan a los obtenidos por Ruiz et al. (2005) en Gmelina arbórea con un 60\% de estacas enraizadas en un sustrato formado por perlita, vermiculita, turba y cáscara de arroz en partes iguales. Esto demuestra la utilidad del sustrato turba, el cual es ideal para la propagación de guayacán, ya que está provisto de suficiente porosidad para permitir una buena 
Cuadro 2. Promedios del efecto simple de los factores, sustrato, hormona ANA y hormona AIB en la propagación asexual de Tabebuia donnell-Smithii (guayacán blanco)

\begin{tabular}{|c|c|c|c|c|c|c|c|}
\hline $\begin{array}{l}\text { Efecto simple de los } \\
\text { tratamientos }\end{array}$ & $\begin{array}{l}\text { Porcentaje de } \\
\text { sobrevivencia }\end{array}$ & $\begin{array}{l}\text { Porcentaje de } \\
\text { enraizamiento }\end{array}$ & $\begin{array}{l}\text { Número de } \\
\text { raíces }\end{array}$ & $\begin{array}{l}\text { Longitud } \\
\text { de raíces }\end{array}$ & $\begin{array}{l}\text { Número } \\
\text { de brotes }\end{array}$ & $\begin{array}{l}\text { Longitud } \\
\text { de brotes }\end{array}$ & Vigor \\
\hline A1.Turba & $84.7 \mathrm{a}$ & $84.7 \mathrm{a}$ & $7.0 \mathrm{a}$ & $6.1 \mathrm{a}$ & $1.2 \mathrm{a}$ & $2.4 \mathrm{a}$ & $3.1 \mathrm{a}$ \\
\hline A2. Arena & $77.1 \mathrm{a}$ & $71.5 \mathrm{~b}$ & $6.3 \mathrm{~b}$ & $2.8 \mathrm{~b}$ & $1.0 \mathrm{~b}$ & $1.2 \mathrm{~b}$ & $1.9 \mathrm{~b}$ \\
\hline B1. ANA $1,500 \mathrm{mg} \mathrm{kg}^{-1}$ & $84.4 \mathrm{a} \mathrm{b}$ & $83.3 \mathrm{a} \mathrm{b}$ & $9.7 \mathrm{a}$ & $4.9 \mathrm{a}$ & $1.1 \mathrm{a} \mathrm{b}$ & $1.7 \mathrm{~b}$ & $2.8 \mathrm{a}$ \\
\hline B2. ANA 2,000 mg kg-1 & $65.6 \mathrm{~b}$ & $64.6 \mathrm{~b}$ & $4.9 \mathrm{~b}$ & $3.5 \mathrm{~b}$ & $1.1 \mathrm{a} \mathrm{b}$ & 1.1 & $1.8 \mathrm{~b}$ \\
\hline B3. Testigo & $92.7 \mathrm{a}$ & $86.5 \mathrm{a}$ & $5.4 \mathrm{~b}$ & $4.9 \mathrm{a}$ & $1.1 \mathrm{a}$ & $2.5 \mathrm{a}$ & $3.0 \mathrm{a}$ \\
\hline C1. AIB $1,500 \mathrm{mg} \mathrm{kg}^{-1}$ & $85.4 \mathrm{a}$ & $84.3 \mathrm{a}$ & $9.6 \mathrm{a}$ & $5.2 \mathrm{a}$ & $1.1 \mathrm{a} \mathrm{b}$ & $2.1 \mathrm{a}$ & $2.8 \mathrm{a}$ \\
\hline C2. AIB 2,000 mg kg-1 & $67.7 \quad b$ & $65.6 \mathrm{~b}$ & $5.5 \mathrm{~b}$ & $3.5 \mathrm{~b}$ & $1.1 \mathrm{a} \mathrm{b}$ & $2.0 \mathrm{a} \mathrm{b}$ & $2.3 \mathrm{~b}$ \\
\hline C3. Testigo & $89.6 \mathrm{a}$ & $84.4 \mathrm{a}$ & $5.0 \mathrm{~b}$ & $4.5 \mathrm{a} \mathrm{b}$ & $1.1 \mathrm{a}$ & $1.4 \mathrm{~b}$ & $2.5 \mathrm{a} \mathrm{b}$ \\
\hline CV $(\%)$ & 13.77 & 15.96 & 15.84 & 14.46 & 9.51 & 13.08 & 10.59 \\
\hline
\end{tabular}

Medias seguidas por la misma letra no presentan diferencias estadísticas (Tukey, $\mathrm{p} \leq 0.05$ ).

aireación y una alta capacidad de retención de agua; tiene un buen drenaje y está libre de patógenos (Hartmann et al., 1992). Coincidiendo también con Van den Heede y Lecourt (1989), quienes mencionan que para favorecer el desarrollo radicular de las microestacas, deben estar colocadas en condiciones favorables.

Por otro lado, las concentraciones 1,500 y $0 \mathrm{mg}$ $\mathrm{kg}^{-1}$ de ANA y AIB promueven un alto porcentaje de enraizamiento, apreciándose que a medida que se aumentó la concentración a 2,000 $\mathrm{mg} \mathrm{kg}^{-1}$ se redujeron los promedios en las variables analizadas, coincidiendo con lo expuesto por Kozlowski et al. (1991) citado por Latsague et al. (2009) quienes mencionan que las auxinas en bajas concentraciones promueven el crecimiento de raíces secundarias y adventicias y en algunas especies las concentraciones inhiben el crecimiento de raíces principales.

Para la variable número de raíces, en el efecto simple se apreciaron diferencias significativas, siendo los mejores promedios los obtenidos en los factores A, B y C los niveles A1 (Turba), B1 (ANA 1,500 mg $\mathrm{kg}^{-1}$ ) y C1 (AIB 1,500 $\mathrm{mg} \mathrm{kg}^{-1}$ ) (Cuadro 2). Gerding et al. (1996) manifiestan que la calificación de la calidad de los sustratos como medio de enraizamiento, además de sus características propias, también depende de la especie que se propague en ellos, estos autores también señalan que para Podocarpus nubigena Lindl, el sustrato de corteza compostada sola o mezclada con otros componentes fue adecuado, en cambio, para el guayacán blanco se considera adecuado el sustrato turba. Lo anterior corrobora lo expresado por Peate (1989) citado por Gerding et al. (1996) quienes mencionan la necesidad de determinar para cada especie su mejor sustrato. La función de los sustratos de cultivo es sustituir al suelo, permitiendo el anclaje y adecuado crecimiento del sistema radicular de la planta. El suelo, factor de producción esencial en la agricultura, actúa como soporte físico de los cultivos y les proporciona los nutrientes, el aire y el agua que precisan. De ello, se desprende la importancia de definir las características físicas, químicas y biológicas de los sustratos de cultivo (Ministerio de Agricultura Alimentación y Medio Ambiente, 2010).

Los resultados obtenidos en esta investigación difieren del promedio obtenido por Moratinos et al. (2008) en Malpighia glabra L. y M. emarginata Sessé \& Moc. ex D.C. con 0.89 a 3.02 raíces respectivamente, quienes mencionan además que estas diferencias se asocian a las técnicas de enraizamiento empleadas (concentración, sustrato, entre otras). También son inferiores a los obtenidos por Tarnowski y Del Castillo (2005) en tres especies de cedro: Cedrela balansae (cedro orán), Toona ciliata (cedro australiano), Cedrela odorata (cedro mexicano), donde no se observó emisiones radiculares en ninguna de las estacas al ser tratadas con diferentes concentraciones ANA.

La longitud de raíces mostró un efecto positivo en el factor A (sustrato turba), este efecto demuestra que la longitud no dependen solo de la aplicación de reguladores de crecimiento, sino que serían otros los factores influyentes en ellas, tales como el tipo de sustrato utilizado (Ram et al. 1993), el pH de éste (Santelices y García 2003) u otros. En el factor B hormona ANA las concentraciones $1,500 \mathrm{mg} \mathrm{kg}^{-1} \mathrm{y}$ testigo, así como en el factor C (AIB) la concentración $1,500 \mathrm{mg} \mathrm{kg}^{-1}$ fueron los más altos con promedios entre 4.52 y $6.07 \mathrm{~cm}$. Estos resultados superan los obtenidos por Latsague et al. (2009) en Eucryphia glutinosa al ser tratados con $500 \mathrm{mg} \mathrm{L}^{-1}$ AIB con un promedio de 3.01 $\mathrm{cm}$ de longitud. En las variables número y longitud de brotes se apreciaron diferencias estadísticas, siendo el sustrato turba el que indujo un efecto positivo para estas 
variables así como la concentración testigo, a excepción de la longitud que fue favorecida en la concentración AIB 1,500 $\mathrm{mg} \mathrm{kg}^{-1}$.

En los datos obtenidos no se observa una tendencia a aumentar la longitud de las raíces a mayor concentración hormonal. Estos resultados difieren de las respuestas obtenidas por Melgares et al. (2002) citado por Latsague et al. (2008) quienes señalan que se observa una tendencia a aumentar los valores de longitud de raíces a mayores temperaturas y mayores concentraciones de AIB en Coriaria myrtifolia Linn. Los resultados obtenidos en longitud son comparables con los descritos por García et al. (2001) en la solanácea Physolis ixocarpa Brot., que en tratamientos con concentración de $1,500 \mathrm{mg} \mathrm{kg}^{-1}$ de AIB incrementan la longitud de las raíces. Lo anterior indica que el uso de reguladores de crecimiento es una forma de mejorar el enraizamiento de diversas especies vegetales.

En esta investigación se observó un efecto estrechamente relacionado entre el vigor de las plantas y las demás variables, probablemente debido a que el proceso de crecimiento y desarrollo está relacionado con los diferentes factores analizados, sobre todo a la cantidad de raíces desarrolladas en los sustratos utilizados, ya que es el medio por el cual la planta toma los nutrientes, los mismos fueron aplicados al inicio al sustrato. Estos resultados concuerdan con lo expresado por Aguilar et al. (2010) quienes mencionan que el vigor es la capacidad que tienen las plantas para crecer y la fuerza para desarrollarse; También corrobora lo expresado por Hartmann et al. (2002) quienes manifiestan que el comportamiento de producción de raíces adventicias es propio para cada especie y puede estar determinado también por factores ambientales como luz, temperatura, fotoperíodo y estado fisiológico de la planta, así como por los reguladores del crecimiento vegetal (Davies, 1995), concentración de oxígeno en el medio radicular (Kozlowski, 1982). El efecto del déficit de oxígeno sobre el crecimiento de las plantas está directamente relacionado con la inhibición del desarrollo radical, produciendo una influencia negativa en el suministro de agua y nutrimentos (Rodríguez y Ramírez, 1987). Las condiciones de estrés es otro factor que influye en el normal desarrollo de la planta, el mismo es definido por Levitt (1980) como: “Cualquier factor ambiental potencialmente desfavorable para los organismos vivos". Santelices (2005) afirma que la capacidad para enraizar está influenciada por la planta madre o donadora de las estacas cosechadas.

Interacciones. El análisis estadístico de las interacciones las variables porcentaje de sobrevivencia y enraizamiento no mostró diferencias significativas para ninguna de las interacciones a excepción de la
$\mathrm{BxC}$ en la concentración (B2: 2,000 mg kg-1 + C2: $2,000 \mathrm{mg} \mathrm{kg}^{-1}$ ) con el promedio más bajo $37.50 \mathrm{y}$ $34.37 \%$ tanto para sobrevivencia como enraizamiento respectivamente, dicho efecto fue similar en las demás variables analizadas (Cuadro 3).

Hartmann et al. (2002), citado por Uribe et al. (2011) señalan que AIB no es tóxico en un amplio rango de concentraciones, sin embargo en esta investigación se observa un efecto negativo a medida que aumenta la concentración hormonal. Esto puede indicar que la dosis óptima de AIB depende de la especie y de la condición fisiológica en que se encuentra la estaca al momento de ser recolectada.

En las interacción $\mathrm{AxB}$, también se observó diferencias significativas con promedios altos en las variables: Numero de raíces (A1 turba por B1 1,500 mg kg-1 ANA) (A2 arena por B1 1,500 mg kg-1 ANA), longitud de raíz y vigor (A1turba por B1 1,500 mg $\mathrm{kg}^{-1}$ ANA) y (A1 turba por B3 testigo) (Cuadro 3). Estos resultados concuerdan con lo manifestado por Kozlowski et al. (1991), citado por Latsague et al. (2009) quienes señalan que la utilización de dosis adecuadas de reguladores de crecimiento es muy importante, puesto que las concentraciones óptimas varían con las especies estudiadas. Por otro lado, Wise y Cadwell (1992), indican que entre las muchas relaciones químicas de hormonas a ser colocadas en las estacas, las más beneficiosas y conocidas son las auxinas, pues estas mejoran la cantidad y calidad de raíces sobres las estacas. Mesén et al. (1997) y Palanisamy et al. (1998), citados por Latsague et al. (2010), señalan que la aplicación de auxinas mejora el desarrollo de las raíces adventicias, demostrando en la presente investigación que las auxinas ANA y AIB a 1,500 mg $\mathrm{kg}^{-1}$ y AIB 1,500 $\mathrm{mg} \mathrm{kg}^{-1}$, son necesarias para tener un buen sistema radicular. Santelices (1993), también afirma que las sustancias más utilizadas para estimular el crecimiento radicular es el ácido indolbutírico y al ácido indolacético los mismos que son compuestos orgánicos sintetizados en una parte de la planta y que se trasloca a otra parte donde a concentraciones adecuadas induce una respuesta fisiológica.

Al analizar las interacciones se observa que la cantidad de raíces así como su longitud no dependen solamente de la aplicación de reguladores de crecimiento, sino que también influyen otros factores tales como el tipo de sustrato utilizado, el pH de éste u otros (Ram et al., 1993, citado por Albany et al., 2004; Santelices y García, 2003). Por otro lado, Tureckaya y Polikarpova (1968), citado por Henselová (2002) mencionan que un efecto positivo de estimulantes en el proceso de enraizamiento de las plantas se logra si las demás condiciones se cumplen, tales como la temperatura óptima de la atmósfera, el tipo y humedad adecuada del 
Cuadro 3. Promedio de las interacciones Factor A (sustratos) por factor B (ANA) por factor C (AIB) en la propagación asexual de Tabebuia donnell-Smithii (guayacán blanco)

\begin{tabular}{|c|c|c|c|c|c|c|c|c|c|c|c|c|}
\hline \multirow{2}{*}{$\begin{array}{c}\text { Interacciones } \\
\text { A1B1 }\end{array}$} & \multirow{2}{*}{$\begin{array}{c}\begin{array}{c}\text { Porcentaje de } \\
\text { sobrevivien- } \\
\text { cia }\end{array} \\
89.58 \mathrm{a}\end{array}$} & \multirow{2}{*}{$\begin{array}{c}\begin{array}{c}\text { Porcentaje } \\
\text { de enraiza- } \\
\text { miento }\end{array} \\
89.58 \mathrm{a}\end{array}$} & \multicolumn{2}{|c|}{$\begin{array}{l}\text { Número de } \\
\text { raíz }\end{array}$} & \multicolumn{2}{|c|}{$\begin{array}{l}\text { Longitud de } \\
\text { raíz }\end{array}$} & \multicolumn{2}{|c|}{$\begin{array}{l}\text { Longitud de } \\
\text { brotes }\end{array}$} & \multicolumn{2}{|c|}{$\begin{array}{l}\text { Número de } \\
\text { brotes }\end{array}$} & \multicolumn{2}{|l|}{ Vigor } \\
\hline & & & $9.41 \mathrm{a}$ & & $6.41 \mathrm{a}$ & & $2.14 \mathrm{a}$ & & $1.16 \mathrm{a}$ & & $3.41 \mathrm{a}$ & \\
\hline A1B2 & $66.66 \mathrm{a}$ & $66.66 \mathrm{a}$ & 5.25 & $\mathrm{~b}$ & 4.60 & $\mathrm{~b}$ & $1.53 \mathrm{a}$ & & $1.08 \mathrm{a}$ & & $2.16 \quad b$ & \\
\hline A1B3 & $97.91 \mathrm{a}$ & $97.91 \mathrm{a}$ & $6.41 \mathrm{a}$ & & $7.20 \mathrm{a}$ & & $3.63 \mathrm{a}$ & & $1.25 \mathrm{a}$ & & $3.75 \mathrm{a}$ & \\
\hline $\mathrm{A} 2 \mathrm{~B} 1$ & $79.16 \mathrm{a}$ & $77.08 \mathrm{a}$ & $10.00 \mathrm{a}$ & & 3.30 & $\mathrm{~b}$ & $1.29 \mathrm{a}$ & & $1.00 \mathrm{a}$ & & $2.16 \quad b$ & \\
\hline A2B2 & $64.58 \mathrm{a}$ & $62.50 \mathrm{a}$ & 4.58 & $\mathrm{~b}$ & 2.40 & $\mathrm{~b}$ & $0.75 \mathrm{a}$ & & $1.08 \mathrm{a}$ & & $1.41 \mathrm{~b}$ & \\
\hline $\mathrm{A} 2 \mathrm{~B} 3$ & $87.50 \mathrm{a}$ & $75.00 \mathrm{a}$ & 4.41 & $\mathrm{~b}$ & 2.61 & $\mathrm{~b}$ & $1.41 \mathrm{a}$ & & $1.00 \mathrm{a}$ & & $2.25 \mathrm{~b}$ & \\
\hline $\mathrm{A} 1 \mathrm{C} 1$ & $89.58 \mathrm{a}$ & $89.58 \mathrm{a}$ & $9.25 \mathrm{a}$ & & $7.32 \mathrm{a}$ & & $2.86 \mathrm{a}$ & & $1.16 \mathrm{a}$ & & $3.50 \mathrm{a}$ & \\
\hline $\mathrm{A} 1 \mathrm{C} 2$ & $72.91 \mathrm{a}$ & $72.91 \mathrm{a}$ & $6.58 a$ & & $4.93 \mathrm{a}$ & & $2.69 \mathrm{a}$ & & $1.25 \mathrm{a}$ & & $2.83 \mathrm{a}$ & \\
\hline $\mathrm{A} 1 \mathrm{C} 3$ & $91.66 \mathrm{a}$ & $91.66 \mathrm{a}$ & $5.25 a$ & & $5.95 \mathrm{a}$ & & $1.75 \mathrm{a}$ & & $1.08 \mathrm{a}$ & & $3.00 \mathrm{a}$ & \\
\hline $\mathrm{A} 2 \mathrm{C} 1$ & $81.25 \mathrm{a}$ & $79.16 \mathrm{a}$ & $9.91 \mathrm{a}$ & & $3.14 \mathrm{a}$ & & $1.24 \mathrm{a}$ & & $1.00 \mathrm{a}$ & & $2.00 \mathrm{a}$ & \\
\hline $\mathrm{A} 2 \mathrm{C} 2$ & $62.50 \mathrm{a}$ & $58.33 \mathrm{a}$ & $4.41 \mathrm{a}$ & & $2.08 \mathrm{a}$ & & $1.24 \mathrm{a}$ & & $0.91 \mathrm{a}$ & & $1.75 \mathrm{a}$ & \\
\hline $\mathrm{A} 2 \mathrm{C} 3$ & $87.50 \mathrm{a}$ & $77.08 \mathrm{a}$ & $4.66 a$ & & $3.09 \mathrm{a}$ & & $0.96 \mathrm{a}$ & & $1.16 \mathrm{a}$ & & $2.08 \mathrm{a}$ & \\
\hline $\mathrm{B} 1 \mathrm{C} 1$ & $78.12 \mathrm{a}$ & $75.00 \mathrm{a}$ & $13.50 \mathrm{a}$ & & $4.94 \mathrm{a}$ & & 1.97 & b c & $1.00 \mathrm{a}$ & $\mathrm{b}$ & $2.87 \mathrm{a} \mathrm{b}$ & \\
\hline $\mathrm{B} 1 \mathrm{C} 2$ & $81.25 \mathrm{a}$ & $81.25 \mathrm{a}$ & 8.25 & $\mathrm{~b}$ & $4.12 \mathrm{a}$ & & 1.98 & $\mathrm{bcd}$ & $1.25 \mathrm{a}$ & $\mathrm{b}$ & $2.87 \mathrm{a} \mathrm{b}$ & \\
\hline $\mathrm{B} 1 \mathrm{C} 3$ & $93.75 \mathrm{a}$ & $93.75 \mathrm{a}$ & 7.37 & $\mathrm{~b}$ & $5.49 \mathrm{a}$ & & 1.20 & d & $1.00 \mathrm{a}$ & $\mathrm{b}$ & $2.62 \mathrm{a} \mathrm{b}$ & \\
\hline $\mathrm{B} 2 \mathrm{C} 1$ & $78.12 \mathrm{a}$ & $78.12 \mathrm{a}$ & 7.62 & $\mathrm{~b}$ & $5.09 a$ & $\mathrm{~b}$ & 1.76 & $\mathrm{c} \mathrm{d}$ & 1.00 & $\mathrm{~b}$ & $2.25 \mathrm{a} \mathrm{b}$ & \\
\hline $\mathrm{B} 2 \mathrm{C} 2$ & $37.50 \quad b$ & $34.37 \quad b$ & 1.75 & $\mathrm{c}$ & 1.00 & $\mathrm{c}$ & 0.48 & e & 0.87 & $\mathrm{c}$ & 1.00 & $c$ \\
\hline $\mathrm{B} 2 \mathrm{C} 3$ & $81.25 \mathrm{a}$ & $81.25 \mathrm{a}$ & 5.37 & $\mathrm{~b}$ & $4.41 \mathrm{a}$ & $\mathrm{b}$ & 1.19 & d & $1.37 \mathrm{a}$ & & $2.12 \mathrm{~b}$ & \\
\hline $\mathrm{B} 3 \mathrm{C} 1$ & $100.00 \mathrm{a}$ & $100 \mathrm{a}$ & 7.62 & $\mathrm{~b}$ & $5.65 \mathrm{a}$ & & 2.44 & $\mathrm{~b}$ & $1.25 \mathrm{a}$ & & $3.12 \mathrm{a}$ & \\
\hline B3C2 & $84.37 \mathrm{a}$ & $81.25 \mathrm{a}$ & 6.50 & $\mathrm{~b}$ & $5.39 \mathrm{a}$ & & $3.44 \mathrm{a}$ & & $1.12 \mathrm{a}$ & $\mathrm{b}$ & $3.00 \mathrm{a} \mathrm{b}$ & \\
\hline $\mathrm{B} 3 \mathrm{C} 3$ & $93.73 \mathrm{a}$ & $78.12 \mathrm{a}$ & 2.12 & $\mathrm{c}$ & 3.66 & $\mathrm{~b}$ & 1.69 & $\mathrm{~b} \mathrm{c} \mathrm{d}$ & $1.00 \mathrm{a}$ & $\mathrm{b}$ & $2.87 \mathrm{a} \mathrm{b}$ & \\
\hline $\mathrm{CV}(\%)$ & 13.77 & 15.96 & 15.8 & & 14.4 & & & 3.08 & 9.51 & & 10.59 & \\
\hline
\end{tabular}

sustrato, además Ruiz et al. (2005) mencionan que el mayor número y tamaño de raíces observados en yemas apicales podrían estar asociados con la mayor velocidad de enraizado y brotación en este tipo de estacas.

Tratamientos. No se observaron diferencias significativas para un nivel de probabilidad de $\mathrm{p} \leq 0.05$ según Tukey entre los distintos tratamientos. Este ensayo evidencia que las variables evaluadas no responden de manera significativa a la aplicación de auxinas y diferentes sustratos en forma combinada, pero si lo hacen de manera aislada o combinada entre dos factores. Resultados similares obtuvieron Hernández et al. (2005); Ruiz et al. (2005) y Latsague et al. (2009) en cuanto a los tratamientos. La homogeneidad en su respuesta puede estar relacionada a que las estacas fueron recolectadas en época lluviosa con suficiente acumulación de auxinas, que le otorga mayor capacidad de los tejidos de sintetizar las auxinas, lo que favoreció el movimiento basipétalo para que las mismas presentaran brotes y enraizaran con facilidad, lo que concuerda con lo expresado por Hernández et al. (2005), Da Fonseca et al. (1991), Hoffmann et al. (1995) y Rossal y Kersten, (1997). También coincide con lo expresado por Hartmann y Kester (1998), citado por Santelices y Cabello (2006), quienes menciona que un factor determinante en estos resultados puede estar relacionado con la época de recolección de las estacas, período de máximo crecimiento vegetativo, en el que las auxinas promueven la formación de raíces adventicias, debido a que las yemas y hojas son grandes productoras de auxinas. Estos resultados sin embargo superan a los obtenidos por Santelices y Bobadilla (1997) en estacas de Peumus boldus con un 15\% de enraizamiento los seis meses. 


\section{Conclusiones}

$\mathrm{E}$ s factible implementar con éxito un programa masivo de propagación clonal de guayacán blanco con estacas juveniles.

En el efecto simple el mejor sustrato fue turba para todas las variables evaluadas a excepción del porcentaje de sobrevivencia que fue indistinto tanto para turba, como para arena. La hormona ANA a $0 \mathrm{mg} \mathrm{kg}^{-1}$ indujo la mejor respuesta excepto para el número de raíces y longitud que se vio favorecida por la concentración $1,500 \mathrm{mg} \mathrm{kg}^{-1}$.

En la interacción $(\mathrm{AxB})$, sustrato turba $(\mathrm{A})$ mostró un efecto positivo en las variables número de raíces, longitud de raíz y vigor al ser tratadas con ANA (B) a 0 y $1,500 \mathrm{mg} \mathrm{kg}^{-1}$.

En la interacción $(\mathrm{BxC})$ los promedios más bajos en todas las variables se observaron con la concentración B2: 2,000 mg kg-1 ANA más C2: 2,000 mg kg-1 AIB.

El mayor número de raíces se obtuvo en la interacción (BxC) $1,500 \mathrm{mg} \mathrm{kg}^{-1}$ ANA y $1,500 \mathrm{mg} \mathrm{kg}^{-1}$ AIB y el mejor vigor de plantas $0 \mathrm{mg} \mathrm{kg}^{-1}$ ANA y 1,500 mg kg-1 AIB.

\section{Literatura CITADA}

Aguilar, M., P. Macario, E. Huerta, S. Hernández, R. de Alba, E. García. 2010. Crecimiento y productividad de chaya (Cnidoscolus chayamansa Mcvaugh, Euphorbiaceae) con densidad de plantación variable. Cultivos tropicales 31(4).

Albany, N., J. Vílchez, Z. Viloria, C. Castro y J. Gadea. 2004. Propagación asexual del guayabo mediante la técnica de acodo aéreo. Agronomía Trop. 54(1): 63-73.

Cruz, N., J. Morante, M. Acosta. 2008. Propagación Vegetativa de Fernan Sánchez (Triplaris guayaquilensis) mediante la utilización de hormonas de enraizamiento (ANA y AIB). Revista Ciencia y Tecnología 1(1):7-10.

Da Fonseca, C., J. Sperandio, M. Fernández, D. Bueno, y R. Lima. 1991. Propagaçao vegetativa do jacaranda da baia (Dalbergia nigra Fr. Allem) através da estaquía. Pesquisa Agropecuaria Brasilera 26(1):31-37.

Davies, P. J. 1995. Plant Hormones: Physiology, Biochemistry and Molecular Biology. Kluwer Academic Publisher, The Netherlands. 883p.

FAO 2010. FAO Forestry, XIII World Forestry Congress. Unasylva. 61(234-235):71-73.

García, L., J. Jiménez, L. Peña y P. Rodríguez. 2001. Propagación vegetativa en tomate de cáscara (Physalis ixocarpa Brot.) mediante enraizamiento de esquejes. Agricultura Técnica México
27(1):27-33.

Gerding, V., M. Hermosilla y R. Grez. 1996. Sustratos de corteza compostada para la propagación vegetativa de estacas de tallo de Podocarpus nubigena Lindl. y Eucryphia cordifolia Cav. Bosque 17(2):57-64.

Gutiérrez, B. y P. Chung. 1994. Propagación vegetativa y silvicultura clonal en eucalipto. Ciencia e Investigación forestal. 8(1).

Hartmann, H. T., D. E. Kester and F. T. Davies. 1992. Plant Propagation. Principles and Practices. Fifth Edition.

Hartmann, H. T., D. E. Kester, F. T. Davies and R. L. Geneve. 2002. Plant propagation: Principles and Practice. 7th ed. Prentice Hall Inc. New Jersey, USA. 880 pp.

Henselová, M. 2002. Synergistic effect of benzolinone with IBA and fungicides on the vegetative propagation of ornamental plants, park, and fruit woody species benzothiazoli. Comenius University, Faculty of Natural Sciences, Department of Plant Physiology, Bratislava, Slovak Republic. Horticultural Science 29(2):41-50.

Hernández, J., H. Aramendiz, C. Cardona. 2005. Influencia del ácido indolbutírico y ácido naftalenoacético sobre el enraizamiento de esquejes de caña flecha (Gynerium sagittatum Aubl.)Temas Agrarios 10(1):5-13.

Hoffmann, A., J. Fachinella y A. Dos Santos. 1995. Propagação de mirtilo (Vaccinium ashei Reade) através de estacas. Pesquisa Agropecuaria Brasileira 30(2):231-236.

John, J. K. 1989. Tabebuia donnell-smithii Rose. SOITF-SM-25. New Orleans, LA: U.S. Department of Agriculture, Forest Service, Southern Forest Experiment Station. 4 p.

Koenig, A., Melchior, G. H. 1978. Propagación vegetative en árboles forestales. INDIRENA/ PNUD/FAO/CONIF. Bogotá, Colombia: Proyecto Investigaciones y Desarrollo Industrial Forestales. COL/74/005. PIF 9.38

Kozlowski, T. T. 1982. Water supply and the tree growth. Part I. Water deficits. For. Abs. 43(2):57-95.

Latsague, V., D. Sáez, B. Hauenstein y F. Peña-Cortés. 2010. Propagación vegetativa de Myrceugenia exsucca y Blepharocalyx cruckshanksii, especies dominantes del bosque pantanoso de la Depresión Intermedia de la región de La Araucanía. Bosque 31(3):247-251.

Latsague, V., D. Sáez y B. Hauenstein. 2008. Inducción de enraizamiento en estacas de Berberidopsis corallina con Acido Indolbutírico. Bosque 29(3): 227-230.

Latsague, V., D. Sáez y D. Yáñez. 2009. Efecto del ácido indolbutírico en la capacidad rizogénica de estacas 
de Eucryphia glutinosa. Bosque 30(2):102-105.

Levitt, J. 1980. Responses of plants to environmental stresses. I. Chilling, freezing, and high temperature stresses. Academic Press, New York. 497 p.

Ministerio de Agricultura Alimentación y Medio Ambiente. 2010. Sustratos de cultivo. (en línea). Consultado 4 dic. 2012. Disponible en: http://www. magrama.gob.es/es/agricultura/temas/medios-deproduccion/sustratos-cultivo/

Ministerio de Relaciones Exteriores, Comercio e Integración. 2010. Medio Ambiente y Desarrollo Sustentable, Manejo Sustentable de los Bosques. (en línea). Consultado 18 Ago. 2010. Disponible en: http://www.mmrree.gob.ec/pol_exterior/ambi ente_bosques.asp

Monteuuis, O., M-C. Bon, DKS. Goh. 1998. Teak propagation by in vitro culture. Bois et Forets des.

Morales, C., J. Calana, J. Corbera, y R. Rivera. 2011. Evaluación de sustratos y aplicación de Hongos micorrízicos arbusculares en Begonia Sp. Cultivos Tropicales 32(1).

Moratinos, P., E. Flores, A. Gomez. 2008. Enraizamiento de estacas de semeruco (Malpighia glabra L. y M. emarginata Sessé \& Moc. ex D.C.). Rev. Fac. Agron. 25(3):405-420.

Mroginski, L. A. y W. M. Roca. 1991. Propagación clonal in vitro. En: Cultivo de tejidos en la agricultura. Fundamentos y aplicaciones. Ed. por William Roca y Luis A. Mroginski. Cali, Colombia. CIAT (Centro Internacional de agricultura Tropical). pp. 19-40.

Murashige, T. and F. Skoog. 1962. A revised medium of rapid growth and bioassays with tobacco tissue. Physiol. Plantarum. 15:473-497.

Navarro, R. M., P. Villar-Salvador, A. del Campo. 2006. Morfología y establecimiento de plantones. In: Cortina, J., Peñuelas, J.L., Puértolas, J., Savé, R., Vilagrosa, A. (Eds). Calidad de planta forestal para la restauración en ambientes mediterráneos. Estado actual de conocimientos. DGB. Ministerio de Medio Ambiente, Serie forestal. Madrid. pp. 67-88.

Ram, S., S. Govind, R. Chandra, S. Govind. 1993. Efficiency of sugar cane and auxins in relation to rooting and survival of guava layers in acid Alfisol. Progressive Horticulture 23(1-4):22-25.

Ramos, L., N. Cruz, J. Morante y O. Villasis. 2006. Empleo de hormonas ANA y AIB estimuladoras de enraizamiento para la propagación vegetativa de Chlorophora tinctoria (L) Gaud (moral fino) en El Litoral Ecuatoriano. Foresta veracruzana 8(1): 9-12.

Rodríguez, B. y R. Ramírez. 1987. Influencia de la tasa de difusión de oxígeno en el suelo sobre la acumulación de materia seca en maíz. Agronomía Tropical 73(4-6):53-62.
Rossal, P. y E. Kersten. 1997. Efeito do ácido indolbutírico no enraizamento de estacas de laranjeira cv. Valência (Citrus sinensis Osbeck) sobcondições intermitentes de nebulização. Scienti Agricola 54(1-2):9-13.

Ruiz, R., J. Vargas, V. Cetina, A. Villegas. 2005. Efecto del ácido indolbutírico (AIB) y tipo de estaca en el enraizado de Gmelina arbórea Roxb: Revista Fitotecnia Mexicana 28(4):319-326.

Santelices, R. 1993. Propagación vegetativa de Raulí, Roble y Coihue a partir de estacas. Ciencia e Investigación Forestal 7(1):37-47.

Santelices, R. 2005. Efecto del árbol madre sobre la rizogénesis de Nothofagus alessandrii. Bosque. 26(3):133-136.

Santelices, R. y A. Cabello. 2006. Efecto del ácido indolbutírico, del tipo de la cama de arraigamiento, del substrato, y del árbol madre en la capacidad de arraigamiento de estacas de Nothofagus glauca (Phil.) Krasser. Revista Chilena de Historia Natural 79:55-64.

Santelices, R. y C. Bobadilla. 1997. Arraigamiento de estacas de Quillaja saponaria Mol. y Peumus boldus Mol. Bosque (Valdivia). 18(2):77-85.

Santelices, R. y C. García. 2003. Efecto del ácido indolbutírico y la ubicación de la estaca en el rebrote de tocón sobre la rizogénesis de Nothofagus alessandrii Espinosa. Bosque 24:53-61.

Serrano, L., y O. Marfa. 1988. Effects of waterlogging on rooting-capacity of cuttings of Hydrangea macrophylla L. Scientia horticulturae. 36:119-224.

Tarnowski, C. y E. del Castillo. 2005. Silvicultura intensiva y desarrollo sustentable con especies valiosas en el noroeste argentino. INTA. (en línea). Consultado 16 Oct. 2012. Disponible inta.gob.ar/... especies.../silvicultura $\% 20 \mathrm{de} \% 20$ especies $\% 20$ valiosas. Tropiques 256:1-11.

Uribe, M., R. Durán, G. Bravo, F. Mora, P. Cartes, C. Delaveau. 2011. Propagación vegetativa de Berberidopsis corallina Hook.f., una especie en peligro de extinción, endémica de Chile. Guyana Botánica. 68(2).

Van den Heede, V. y M. Lecourt. 1989. El Estaquillado: Guía práctica de manipulación de plantas. Editorial Mundi_Prensa. Madrid. España 197 p.

Vozzo, J.A. 2010 . Tropical tree seed manual. Washington DC, USDA Forest Service.

Wendling, I. 2004. Propagación vegetativa de Yerba mate (LLex paraguariensis Saint Hilaire): Estado del arte, tendencias futuras. Embrapa Florestas Editorial CIP. Brasil $42 \mathrm{p}$.

Wise, F. and T. Calwell. 1992. Macropropagation Of Conifers By Stem Cuttings; Aplications Of Vegetative Propagation In Forestry, Southern 
Carranza et al.

Regional Information Exchange Group Biennial Symposium On Forest Genetics. Huntsville Alabama New Orleans Lousinia USDA. Forest Service. Southern Forest Esperiment Stataion: 5173. 